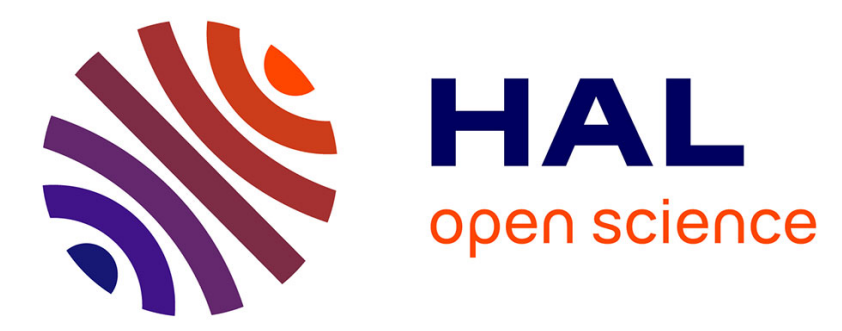

\title{
An inverse COnvolution MEthod for wavenumber extraction (INCOME): Formulations and applications
}

Régis F. Boukadia, Claus Claeys, Christophe Droz, Mohamed Ichchou, Wim Desmet, Elke Deckers

\section{- To cite this version:}

Régis F. Boukadia, Claus Claeys, Christophe Droz, Mohamed Ichchou, Wim Desmet, et al.. An inverse COnvolution MEthod for wavenumber extraction (INCOME): Formulations and applications. Journal of Sound and Vibration, 2021, pp.116586. 10.1016/j.jsv.2021.116586 . hal-03420782

\section{HAL Id: hal-03420782 https://hal.science/hal-03420782}

Submitted on 9 Nov 2021

HAL is a multi-disciplinary open access archive for the deposit and dissemination of scientific research documents, whether they are published or not. The documents may come from teaching and research institutions in France or abroad, or from public or private research centers.
L'archive ouverte pluridisciplinaire HAL, est destinée au dépôt et à la diffusion de documents scientifiques de niveau recherche, publiés ou non, émanant des établissements d'enseignement et de recherche français ou étrangers, des laboratoires publics ou privés. 


\title{
An INverse COnvolution MEthod for wavenumber extraction (INCOME): formulations and applications
}

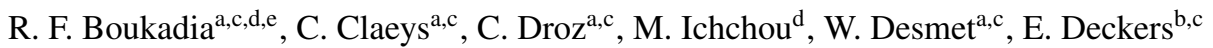 \\ ${ }^{a}$ KU Leuven, Department of Mechanical Engineering, Division LMSD, Celestijnenlaan 300 - box 2420, Heverlee, Belgium \\ ${ }^{b}$ KU Leuven, Campus Diepenbeek, Department of Mechanical Engineering, Wetenschapspark 27, 3590 Diepenbeek, Belgium \\ ${ }^{c}$ DMMS lab, Flanders Make \\ ${ }^{d}$ Ecole Centrale de Lyon, 36 Avenue Guy de Collongue, 69134 Ecully Cedex, France \\ e SIM M3 program, Technologiepark 48, B-9052 Zwijnaarde, Belgium
}

\begin{abstract}
This paper deals with the extraction of wave features in elastic media. An inverse approach is proposed for the identification of wave dispersion characteristics (e.g. k-space) in one- and two-dimensional structures (1D, 2D). The proposed method is similar to the ESPRIT algorithm and the Prony series method and can be considered as an extension of the latter, specifically when applied to $1 \mathrm{D}$ problems. By using a convolution framework, the method is extended to the 2D case for which it allows the estimation of the full k-space by solving a linear problem. The method is called INverse COnvolution MEthod (INCOME). The formulation of INCOME is first detailed and mathematically justified. Both the 1D and 2D cases are detailed and explained. Then several examples are presented for assessing the validity domain of INCOME. These numerical tests clearly show the relevance of INCOME for structured inputs with periodic characteristics.
\end{abstract}

Keywords: Wavenumber Extraction, Dispersion Curves, Inverse Method, Periodic Structures, Wave Propagation, One-dimensional vibroacoustics, Two-dimensional vibroacoustics, Waveguides.

\section{Introduction}

Wavenumber extraction is used in many applications among several domains including: high resolution direction of arrival estimation, protection of electrical power lines [1] and more broadly, electronic surveillance measures. In the context of vibroacoustics, wavenumber extraction provides insights into the wave propagation features of given media [2], can be used for model updating purposes [3] and also for the characterization of periodic structures [4]. Because of its wide range of applications, a number of wavenumber extraction methods have been developed in the literature $[1,3,5-10]$. Most of these methods can extract wavenumbers accurately and consistently when dealing with one dimensional media but difficulties arise when considering wave propagation in two (or more) dimensions. The main issue is that waves propagating in different directions may have different wavenumbers. Consequently, wavenumbers need to be simultaneously retrieved for every possible direction of propagation, forming (in the wavenumber domain) what is called the k-space of a medium (see e.g. Figure 6). This paper aims to address this issue by proposing an efficient method to retrieve the $\mathrm{k}$-spaces of $2 \mathrm{D}$ media. The following paragraphs of this section briefly present the most popular wavenumber extraction methods of the literature. A simple classification of these methods is then established and enables a clear positioning of the proposed method with respect to the state of the art.

In the open literature, Estimation of Signal Parameters via Rotational Invariance Techniques (ESPRIT) was used for wavenumber extraction. The ESPRIT algorithm [6] exploits properties of exponential functions and subspaces spanned by them. Namely, these subspaces are invariant under any translation operator and the exponential functions spanning these subspaces diagonalize the operators. Assuming periodic sampling of the signal, a robust discretized representation of the subspace is built using the Singular Value Decomposition (SVD) on a Hankel matrix of the

Email address: regis. boukadia@kuleuven. be (R. F. Boukadia) 
signal and by keeping only the left-singular vectors corresponding to the signal space (as opposed to the noise space). From this matrix representation (of the subspace), a discretized version of the translation operator corresponding to the sampling period is built by solving a linear least-squares problem. Finally, the propagation constants are obtained by computing the eigenvalues of the operator. The ESPRIT algorithm works in the 1D case and can be applied to the 2D case if the signal of interest can be approximated as the sum of a small number of plane waves. In some cases, the second condition can be artificially forced by partitioning the initial domain of measurement into several subdomains [11].

Like the ESPRIT algorithm, the Prony series method [5] requires periodic sampling of the signal. Assuming there is no noise, the sequence of measurements should be a sum of geometric sequences and satisfy a linear recurrence relationship. An estimate of that relationship's coefficients is obtained by solving a least-squares problem and the propagation constants are obtained by computing the roots of the associated (characteristic) polynomial [12, Ch 2]. This method only works for $1 \mathrm{D}$ cases.

In the frame of already published works related to wavenumber extraction techniques, McDaniel's method [7] is conceptually the simplest among all suggested methods and consists in a nonlinear least-squares fit of the measured signal $U$ by a sum of exponential functions:

$$
U(x)=\sum_{l=1}^{n_{w}} A_{l} \mathrm{e}^{-\mathrm{i} k_{l} x} .
$$

Unlike the ESPRIT algorithm (and the Prony series method), McDaniel's method does not come with specific sampling constraints and is compatible with sparse and scattered measurements. However, it is only applicable in the 1D case.

The Inhomogenous Wave Correlation and the Inverse Wave Decomposition were also considered in the open literature. Both methods were developed to perform 2D wavenumber extraction and assume the following form for the measured signal:

$$
U(x, y)=\int_{0}^{2 \pi} \sum_{l=1}^{n_{w}} A_{l}(\theta) \mathrm{e}^{-\mathrm{i} k_{l}(\theta) \cos (\theta) x} \mathrm{e}^{-\mathrm{i} k_{l}(\theta) \sin (\theta) y} d \theta,
$$

with $n_{w}$ the number of wave types, $\theta$ the angle of propagation, $k_{l}(\theta)$ the $l^{\text {th }}$ wave's wavenumber and $A_{l}(\theta)$ the associated wave amplitude. With this assumption as a basis, the IWC estimates the values of $k_{l}(\theta)$ separately by maximizing the correlation between the displacement field and a plane wave of unknown wavenumber propagating in the direction given by $\theta$. The corresponding assumptions are that $n_{w}=1$ and that $2 \mathrm{D}$ exponential functions with different directions of propagation are orthogonal. Nonetheless, an extension of the IWC dealing with $n_{w}>1$ was proposed in [13] for 1D wave propagation. By contrast, the IWD achieves recovery of the k-space by fitting the displacement field with a discretized version of Eq. (2) and does not require additional assumptions. Because of its underlying assumptions, the IWC typically only gives good results when the characteristic wavelength of the signal is small compared to the size of the domain of measurement. Conversely, the IWD works well when the sampled signal is isotropic and its characteristic wavelength is not too small compared to the size of the domain of measurement. Outside of these conditions, the IWD's least-squares problem is practically unsolvable.

The general properties of all aforementioned methods are summarized in Table 1 wherein the 'Exact' column refers to the ability to extract exact wavenumbers when noiseless data is used. From Table 1, it seems natural to partition

\begin{tabular}{|c|c|c|c|c|c|}
\hline Method & Cases & Exact & Diffuse field & Solver & Sampling Constraints \\
\hline IWC & 1D/2D & No & No & Non-linear Non-convex & None \\
\hline McDaniel & 1D & Yes & No & Non-linear Non-convex & None \\
\hline IWD & 2D & Yes & Yes & Non-linear Non-convex & None \\
\hline Prony Series & 1D & Yes & Yes & Linear & Periodicity \\
\hline ESPRIT & 1D/2D & Yes & No & Linear & Periodicity \\
\hline
\end{tabular}

Table 1: General properties of different wavenumber extraction methods

the aforementioned methods in two classes. The first class contains methods that leverage the algebraic properties of the measured signal in order to extract its characteristics. These methods use well posed linear problems but require 
periodic sampling of the signal of interest. Methods of the second class do not have specific sampling constraints and can manage scattered data. On the other hand, these methods require solving nonlinear and generally non-convex optimization problems in order to provide wavenumber estimates. While several of the methods mentioned work well in the $1 \mathrm{D}$ case, all have strong limitations in the $2 \mathrm{D}$ one. Indeed, the linear methods mentioned above do not have appropriate 2D formulations and even though 2D versions of the non linear methods exists, they come with limitations. Specifically, the IWC only works well when one wave type is present and the modal density is high whereas the IWD only works well at low frequency because its signature optimization problem becomes practically unsolvable in the mid-high frequency range. In this paper, a novel formulation trying to address some of these issues is presented. The highlights of the proposed methods are the following:

- The method relies on solving small linear problems and is exact in both 1D and 2D cases.

- In the 2D case, all properties of the k-space are retrieved together in a coherent manner.

- The proposed method draws inspiration from the Wave Finite Element Method (WFEM) framework [14-17] and can be considered an inverse WFEM.

The rest of this paper is structured as follows. In Section 2, the 1D version of the proposed method is presented. It is the version in which it is closest to the Prony series method. The formulation is derived starting with an unbounded media and considering then a bounded domain. The case of multi-modal wave propagation is also dealt with as an extension. Making use of the convolution formalism, the 2D version of the method is derived in Section 3 where a mathematical justification by comparison to the WFEM is given and the k-space extraction algorithm is detailed. Numerical examples are studied in Section 4. Several cases are considered for the sake of validation of the INCOME formulation. Most examples are inspired from vibroacoustics with or without damping mechanisms. Finally, Section 5 presents possible improvements and use cases of this work and summarizes the main conclusions.

\section{Inverse Convolution Method in one dimension (INCOME 1D)}

In this Section, the 1D version of the proposed INverse COnvolution MEthod (INCOME) is presented. First, INCOME's main principle is derived in subsection 2.1 for a signal composed of a single exponential function. A recurrence relationship is established between successive measurements and reframed in the convolution framework which gives the method its name. In subsection 2.2, the wavenumber domain symmetry of (most) vibroacoustic systems is introduced, hence the signal considered is composed of two exponential functions with opposite wavenumbers. This symmetry is accounted for in INCOME's formulation such that both wavenumbers are retrieved by estimating a single parameter. The general case where many wavenumber pairs are present is tackled in subsection 2.3 and a regularization technique enforcing the wavenumber symmetry to numerical precision is derived in subsection 2.4. Lastly, subsection 2.5 discusses how the number of independent exponential functions present in the signal can be estimated when it is not known in advance.

\subsection{Fundamental principle}

In this subsection, the principle behind the proposed methodology is presented by considering the propagation of a single plane wave on a finite $1 \mathrm{D}$ domain. The size of the domain is $L_{\max }$ and it is assumed to occupy the space region $x \in\left[0, L_{\max }\right]$. The displacement field is represented by a function $U$ of the form:

$$
U(x)=A \mathrm{e}^{-\mathrm{i} k x} .
$$

In Eq. (3), $k$ is a complex wavenumber and $A$ is the complex wave amplitude. Both are unknown but the aim is only to retrieve $k$. To that end, the wavefield $U$ is sampled at $N$ points $\left(x_{1}, \ldots, x_{N-1}, x_{N}\right)$ periodically spaced with distance $L:$

$$
\left\{\begin{array}{l}
x_{n}=x_{1}+(n-1) L \\
U_{n}=U\left(x_{n}\right)+\epsilon_{n}
\end{array} .\right.
$$


In Eq. (4), $x_{n}$ is the abscissa of the $n^{\text {th }}$ measurement point while $\epsilon_{n}$ is the measurement error for the $n^{\text {th }}$ point. In case there is no error, the sequence $\left(U_{n}\right)_{n \in \llbracket 1, N \rrbracket}$ is a geometric sequence with common ratio $\lambda=\mathrm{e}^{-\mathrm{i} k L}$, also called propagation constant, which can be computed by taking the ratio of any two successive measurements.

$$
\lambda=\frac{U_{n+1}}{U_{n}}
$$

The wavenumber $k$ is subsequently retrieved from $\lambda$.

$$
k=\frac{\mathrm{i}}{L} \ln (\lambda) .
$$

In most cases however, the measurements are not perfect, hence only an estimate of the propagation constant can be computed. The most intuitive way to do so is to average the ratios of consecutive terms of the sequence:

$$
\hat{\lambda_{1}}=\frac{1}{N-1}\left(\sum_{n=1}^{N-1} \frac{U_{n+1}}{U_{n}}\right) \text {. }
$$

Eq. (7) leverages all the measurements to form a robust estimate of the propagation constant. However, it cannot be generalized to cases in which several propagation constants must be retrieved from one set of measurements. Consequently, a method easier to generalize is proposed. When no error is present in the measurements, the sequence $\left(U_{n}\right)_{n \in \llbracket 1, N \rrbracket}$ satisfies:

$$
\forall n \in \llbracket 1, N-1 \rrbracket, \quad U_{n+1}-\lambda U_{n}=0 .
$$

To form an estimate of $\lambda$ using this relationship, it is helpful to see it as a convolution product between two sequences. First, the convolution product of two infinite sequences is defined. For two infinite sequences $\left(g_{n}\right)_{n \in \mathbb{Z}}$ and $\left(h_{n}\right)_{n \in \mathbb{Z}}$ their convolution product $\left(e_{n}\right)_{n \in \mathbb{Z}}$ is defined as:

$$
\left\{\begin{array}{l}
\mathbf{e}=\mathbf{g} * \mathbf{h}=\mathbf{h} * \mathbf{g} \\
\forall n \in \mathbb{Z}, e_{n}=\sum_{l=-\infty}^{\infty} g_{n-l} h_{l} .
\end{array}\right.
$$

This definition can be extended to the case of finite sequences. Considering two finite sequences $\left(g_{n}\right)_{n \in \llbracket 1, N \rrbracket}$ and $\left(h_{n}\right)_{n \in \llbracket 1, M \rrbracket}$ such that $M \leq N$ their convolution product $\left(e_{n}\right)_{n \in \llbracket 1, N+1-M \rrbracket}$ is defined by:

$$
\left\{\begin{array}{l}
\mathbf{e}=\mathbf{g} * \mathbf{h}=\mathbf{h} * \mathbf{g} \\
\forall n \in \llbracket 1, N+1-M \rrbracket, e_{n}=\sum_{l=1}^{M} g_{n+M-l} h_{l}
\end{array} .\right.
$$

In this context, Eq. (8) can be rewritten as $\boldsymbol{U} *(1,-\lambda)=0$, therefore, a good estimate of $\lambda$ can be found by least-squares optimization:

$$
\left\{\begin{array}{l}
(\alpha, \beta)=\operatorname{argmin}\left\{\|\boldsymbol{U} *(a, b)\|_{2},\|(a, b)\|_{2}=1\right\} \\
\hat{\lambda}=-\frac{\beta}{\alpha}
\end{array} .\right.
$$

The first part of Eq. (11) seeks to find a two-term sequence that produces a new sequence of minimal norm when convoluted with the discretized displacement field. The constraint $\|(a, b)\|_{2}=1$ is imposed because the norm of the product scales linearly with that of each sequence involved. As a result, making comparisons at equal norms is necessary which means $\alpha$ cannot be chosen equal to 1 . Consequently, $\hat{\lambda}$ is defined by the ratio of $\beta$ and $\alpha$.

Finally, an efficient way of solving of Eq. (11) is proposed by identifying a finite sequence of $n$ terms with the corresponding vector in $\mathbb{C}^{N}$ and casting convolution products as a matrix-vector products. For a sequence $\left(U_{n}\right)_{n \in \llbracket 1, N \rrbracket}$, the matrix $\mathbf{C}_{i}(U)$ is defined as follows:

$$
\mathbf{C}_{i}(U)=\left(\begin{array}{cccc}
U_{i} & U_{i-1} & \ldots & U_{1} \\
U_{i+1} & U_{i} & \ldots & U_{2} \\
\vdots & \vdots & \vdots & \vdots \\
U_{N} & U_{N-1} & \ldots & U_{N+1-i}
\end{array}\right) .
$$


With this notation, the convolution product of Eq. (11) can be rewritten as

$$
\mathbf{U} *(a, b)=\mathbf{C}_{2}(U)\left(\begin{array}{l}
a \\
b
\end{array}\right)=\left(\begin{array}{cc}
U_{2} & U_{1} \\
U_{3} & U_{2} \\
\vdots & \vdots \\
U_{N-1} & U_{N-2} \\
U_{N} & U_{N-1}
\end{array}\right)\left(\begin{array}{l}
a \\
b
\end{array}\right) .
$$

The system of Eq. (11) is finally rewritten using matrices and vectors,

$$
\left\{\begin{array}{l}
\left(\begin{array}{l}
\alpha \\
\beta
\end{array}\right)=\operatorname{argmin}\left\{\mathbf{x}^{*} \mathbf{C}_{2}^{*}(U) \mathbf{C}_{2}(U) \mathbf{x},\|\mathbf{x}\|_{2}^{2}=1\right\} \\
\hat{\lambda}=-\frac{\beta}{\alpha}
\end{array},\right.
$$

and can be solved by computing the eigenvalues and eigenvectors of a positive Hermitian matrix. Indeed, $\alpha$ and $\beta$ are the first and second components of the eigenvector of $\mathbf{D}_{2}(U)=\mathbf{C}_{2}^{*}(U) \mathbf{C}_{2}(U)$ associated to its smallest eigenvalue (see Courant-Fischer-Weyl min-max principle or "min-max" theorem).

\subsection{Introducing a reflected wave}

In most cases, the wave field is not as simple a single plane wave propagating in one direction. Due to the boundaries of the domain, a reflected wave with the opposite wavenumber should also be part of the wave field. In that case, the displacement field takes the form:

$$
U(x)=A \mathrm{e}^{-\mathrm{i} k x}+B \mathrm{e}^{\mathrm{i} k x} .
$$

Consequently, the general form for $\left(U_{n}\right)_{n \in \llbracket 1, N \rrbracket}$, the sequence of measurements, is

$$
\left\{\begin{array}{l}
U_{n}=A \lambda^{n}+B \lambda^{-n}+\epsilon_{n} \\
\lambda=\mathrm{e}^{-\mathrm{i} k L}
\end{array} .\right.
$$

Because the sequence is the sum of two geometric sequences, it satisfies a recurrence relation whose characteristic polynomial (see [12, Ch 2]) is

$$
P_{U}=(X-\lambda)\left(X-\frac{1}{\lambda}\right)=X^{2}-\left(\lambda+\frac{1}{\lambda}\right) X+1 .
$$

Noting $\mu=\lambda+\frac{1}{\lambda}$, the recurrence relationship for the sequence $U$ (in case the error is null) is derived from the coefficients of $P_{U}[12, \mathrm{Ch} 2]$ :

$$
\forall n \in \llbracket 1, N-2 \rrbracket, \quad U_{n+2}-\mu U_{n+1}+U_{n}=0 .
$$

Or, in a better way:

$$
\forall n \in \llbracket 1, N-2 \rrbracket, \quad\left(U_{n+2}+U_{n}\right)-\mu U_{n+1}=0 .
$$

Equations (18) and (19) can also be understood as convolution product between a symmetric (three term long) sequence and the displacement field. An estimate $\hat{\mu}$ of $\mu$ can thus be obtained by solving the following least-squares problem:

$$
\left\{\begin{array}{l}
(\alpha, \beta, \alpha)=\operatorname{argmin}\left\{\|\mathbf{U} *(a, b, c)\|_{2},\|(a, b, c)\|_{2}=1, c=a\right\} \\
\hat{\mu}=-\frac{\beta}{\alpha}
\end{array} .\right.
$$

Estimates for the pair of propagation constants are then obtained as

$$
\left(\hat{\lambda}, \frac{1}{\hat{\lambda}}\right)=\left(\frac{\hat{\mu}+\sqrt{\hat{\mu}^{2}-4}}{2}, \frac{\hat{\mu}-\sqrt{\hat{\mu}^{2}-4}}{2}\right) .
$$


In Eq. (22), the convolution product is put in matrix form similarly to Eq. (13):

$$
\mathbf{U} *(a, b, a)=\mathbf{H}_{1}(U)\left(\begin{array}{l}
a \\
b
\end{array}\right)=\left(\begin{array}{cc}
U_{3}+U_{1} & U_{2} \\
U_{4}+U_{2} & U_{3} \\
\vdots & \vdots \\
U_{N-1}+U_{N-3} & U_{N-2} \\
U_{N}+U_{N-2} & U_{N-1}
\end{array}\right)\left(\begin{array}{l}
a \\
b
\end{array}\right) .
$$

Also a new matrix $\mathbf{A}_{1}$ is introduced,

$$
\mathbf{A}_{1}=\left(\begin{array}{ll}
2 & 0 \\
0 & 1
\end{array}\right)
$$

which accounts for the fact that

$$
\|(a, b, a)\|_{2}^{2}=\left(\begin{array}{l}
a \\
b
\end{array}\right)^{*} \mathbf{A}_{1}\left(\begin{array}{l}
a \\
b
\end{array}\right) .
$$

The system of Eq. (20) is then rewritten using matrices and vectors:

$$
\left\{\begin{array}{l}
\left(\begin{array}{l}
\alpha \\
\beta
\end{array}\right)=\operatorname{argmin}\left\{\mathbf{x}^{*} \mathbf{H}_{1}^{*}(U) \mathbf{H}_{1}(U) \mathbf{x}, \mathbf{x}^{*} \mathbf{A}_{1} \mathbf{x}=1\right\} \\
\hat{\mu}=-\frac{\beta}{\alpha}
\end{array} .\right.
$$

Finally, Eq. (25) is solved by computing the eigenvector associated to the smallest eigenvalue $\rho$ of the following generalized eigenvalue problem:

$$
\left(\mathbf{H}_{1}^{*}(U) \mathbf{H}_{1}(U)\right) \mathbf{x}=\rho \mathbf{A}_{1} \mathbf{x} .
$$

\subsection{General formulation}

Now, the general case is considered. The displacement field is comprised of multiple planewaves and their reflections:

$$
U(x)=\sum_{p=1}^{n_{w}} A_{p} \mathrm{e}^{-\mathrm{i} k_{p} x}+B_{p} \mathrm{e}^{\mathrm{i} k_{p} x} .
$$

Consequently, the general form for $\left(U_{n}\right)_{n \in \llbracket 1, N \rrbracket}$, the sequence of measurements, is:

$$
\left\{\begin{array}{l}
U_{n}=\sum_{p=1}^{n_{w}} A_{p} \lambda_{p}^{n}+B_{p} \lambda_{p}^{-n}+\epsilon_{n} \\
\forall p \in \llbracket 1, n_{w} \rrbracket, \lambda_{p}=\mathrm{e}^{-\mathrm{i} k_{p} L}
\end{array} .\right.
$$

Since $\left(U_{n}\right)_{n \in \llbracket 1, N \rrbracket}$ is a sum a geometric sequences, its characteristic polynomial (see [12, Ch 2]) is

$$
P_{U}=\prod_{p=1}^{n_{w}}\left(X-\lambda_{p}\right)\left(X-\frac{1}{\lambda_{p}}\right) .
$$

Because the propagation constants come in pairs $\left(\lambda_{p}, \frac{1}{\lambda_{p}}\right), P_{U}$ is a palindromic polynomial of even degree:

$$
\left\{\begin{array}{l}
P_{U}=\sum_{p=0}^{2 n_{w}} a_{p} X^{p} \\
\forall p \in \llbracket 0,2 n_{w} \rrbracket, a_{2 n_{w}-p}=a_{p}
\end{array}\right.
$$

and can be rewritten as

$$
P_{U}=a_{n_{w}} X^{n_{w}}+\sum_{p=0}^{n_{w}-1} a_{p}\left(X^{p}+X^{2 n_{w}-p}\right)
$$


The corresponding recurrence relationship for the sequence $U$ is then derived.

$$
\forall n \in \llbracket 1, N-2 n_{w} \rrbracket, \quad a_{n_{w}} U_{n+n_{w}}+\sum_{p=0}^{n_{w}-1} a_{p}\left(U_{n+p}+U_{n+2 n_{w}-p}\right)=0 .
$$

As in the previous cases, an estimate of the coefficients $a_{p}$ is obtained by solving a least-squares problem. This least-squares problem comes from understanding Eq. (32) as a convolution product. First, two matrix families are introduced starting with the family $\mathbf{A}_{n_{w}}$ :

$$
\mathbf{A}_{n_{w}}=\left(\begin{array}{cccc}
2 & 0 & \ldots & 0 \\
0 & 2 & 0 \ldots & 0 \\
\vdots & \ldots & 2 & \ldots \\
0 & \ldots & \ldots & 1
\end{array}\right) .
$$

For all natural integers, $\mathbf{A}_{n_{w}}$ is a $\left(n_{w}+1\right) \times\left(n_{w}+1\right)$ diagonal matrix. Its first $n_{w}$ diagonal terms are equal to 2 while the last one is equal to 1. Like $\mathbf{A}_{1}$, introduced in Eq. (23), $\mathbf{A}_{n_{w}}$ can be understood as weighing in the least-squares problem to account for the fact that most coefficients appear two times in the recurrence relationship of Eq. (32). The second matrix family to be introduced is $\mathbf{H}_{n_{w}}(U)$. It represents the matrix form of the convolution product of a symmetric sequence of $2 n_{w}+1$ terms with the sequence of measurements.

$$
\mathbf{H}_{n_{w}}(U)=\left(\begin{array}{ccccc}
U_{2 n_{w}+1}+U_{1} & U_{2 n_{w}}+U_{2} & \ldots & U_{n_{w}+2}+U_{n_{w}} & U_{n_{w}+1} \\
U_{2 n_{w}+2}+U_{2} & U_{2 n_{w}+1}+U_{3} & \ldots & U_{n_{w}+3}+U_{n_{w}+1} & U_{n_{w}+2} \\
\vdots & \vdots & \ldots & \vdots & \vdots \\
U_{N-1}+U_{N-2 n_{w}-1} & U_{N-2}+U_{N-2 n_{w}} & \ldots & U_{N-n_{w}}+U_{N-n_{w}-2} & U_{N-n_{w}-1} \\
U_{N}+U_{N-2 n_{w}} & U_{N-1}+U_{N-2 n_{w}+1} & \ldots & U_{N-n_{w}+1}+U_{N-n_{w}-1} & U_{N-n_{w}}
\end{array}\right) .
$$

As in the previous subsections, the coefficients of $P_{U}$ are obtained by computing the eigenvector associated to the smallest eigenvalue $\rho$ of the following eigenvalue problem:

$$
\left(\mathbf{H}_{n_{w}}^{*}(U) \mathbf{H}_{n_{w}}(U)\right) \mathbf{x}=\rho \mathbf{A}_{n_{w}} \mathbf{x} .
$$

Finally, the propagation constants' estimates are obtained by finding the roots of the polynomial $P_{U}$.

\subsection{Palindromic transformation}

Due to numerical issues, the computed solutions of $P_{U}(X)=0$ may violate the relationship between the pairs of propagation constants $\left(\lambda_{i}, \frac{1}{\lambda_{i}}\right)$ when a naive root finding algorithm is used. Consequently, a root finding technique enforcing that relationship to numerical precision is presented. First, a rational fraction $Q$ is defined:

$$
Q(X)=\frac{P_{U}(X)}{X^{n_{w}}}=a_{n_{w}}+\sum_{p=0}^{n_{w}-1} a_{p}\left(X^{n_{w}-p}+X^{p-n_{w}}\right) .
$$

The change of variables $Y=X+\frac{1}{X}$ is then performed (for $Q$ ) and yields a polynomial $R$ of degree $n_{w}$ whose roots are $\mu_{i}=\left(\lambda_{i}+\frac{1}{\lambda_{i}}\right)$. The roots $\mu_{i}$ can be computed by applying any classical root finding algorithm on $R$ after which the pairs of propagation constants can be recovered.

$$
\left(\lambda_{i}, \frac{1}{\lambda_{i}}\right)=\left(\frac{\mu_{i}+\sqrt{\mu_{i}^{2}-4}}{2}, \frac{\mu_{i}-\sqrt{\mu_{i}^{2}-4}}{2}\right) .
$$

The proposed root finding method is similar to the transformation of quadratic palindromic eigenvalue problems that appear in optimal control theory and for the 1D WFEM [18]. 


\subsection{Determining the number of waves}

Before determining what the propagation constants and wavenumbers are, it is necessary to know how many waves are present in the wavefield. That number may be known a priori using known physical or numerical proprieties of the phenomenon generating the signal. When that is not the case, that number may be determined by using a stability diagram as in [3] but an alternative method is proposed here. Indeed, the number of waves, $n_{w}$, can be determined by looking at the rank, $r_{n}$, of the matrix $\mathbf{H}_{n}(U)$. As long as $n_{w} \leq n, r_{n}$ should be equal to $n_{w}$. Theoretically, $r_{n}$ is equal to the number of non-zero singular values of $\mathbf{H}_{n}(U)$ and can be directly determined by computing them. However, due to numerical precision and experimental error, singular values below a certain threshold should be considered null when determining $r_{n}$. Procedures for appropriate truncation critera can be found in $[19,20]$. In general the truncation value $S_{t}$ should at least verify $S_{t} \geq \max \left(\alpha S_{\max }, \frac{S_{\max }}{n r}\right)$ with $\alpha$ the numerical precision, $n r$ the signal to noise ratio and $S_{\max }$ the lagerst singular value of $\mathbf{H}_{n}(U)$.

\section{Inverse Convolution Method in two dimensions (INCOME 2D)}

This section deals with the 2D version of INCOME. In subsection 3.1, the 2D version of INCOME is intuitively derived using the convolution framework and partially justified by comparison to the 2D Wave Finite Element Method (WFEM). Finally, subsection 3.2 details how to extract the proper k-space corresponding to a given convolution operator.

\subsection{General version of the $2 D$ case}

The 1D version of the proposed method works by finding a (symmetric) convolution kernel that results in a null sequence when multiplied with a sequence of (periodically spaced) measurements. An intuitive way to extend the method to the 2D case is to search for a 2D symmetric convolution kernel that would do the same with a 2D sequence of measurements. Two questions are raised. Firstly, is it possible to mathematically justify this approach? Secondly, what would be the size of such a convolution kernel and which among the many forms of 2D symmetry should it have? Both questions are partially answered when looking at the 2D Wave Finite Element Method when written as the explicit assembly of a 2D periodic medium composed of identical unit cells (see Appendix A) [21-23]:

$$
\begin{aligned}
\mathbf{F}_{r, s}= & \left(\mathbf{D}_{11}+\mathbf{D}_{22}+\mathbf{D}_{33}+\mathbf{D}_{44}\right) \boldsymbol{\Psi}_{r, s}+ \\
& \left(\mathbf{D}_{13}+\mathbf{D}_{24}\right) \boldsymbol{\Psi}_{r, s+1}+\left(\mathbf{D}_{12}+\mathbf{D}_{34}\right) \boldsymbol{\Psi}_{r+1, s}+\left(\mathbf{D}_{31}+\mathbf{D}_{42}\right) \boldsymbol{\Psi}_{r, s-1}+\left(\mathbf{D}_{21}+\mathbf{D}_{43}\right) \boldsymbol{\Psi}_{r-1, s}+ \\
& \mathbf{D}_{14} \Psi_{r+1, s+1}+\mathbf{D}_{23} \boldsymbol{\Psi}_{r-1, s+1}+\mathbf{D}_{32} \Psi_{r+1, s-1}+\mathbf{D}_{41} \Psi_{r-1, s-1} .
\end{aligned}
$$

In Eq. (38), setting all external forces, $\mathbf{F}_{r, s}$, to zero and applying the Floquet-Bloch theorem leads to the the classical form of the 2D WFEM. Another way of approaching Eq. (38) is to consider it as a convolution product between a 2D sequence of vectors (representing structural displacements) and a 2D sequence of matrices (representing the partial differential equation governing the periodic medium). When no forces are present, this convolution product should result in a null sequence. These observations validate the general concept proposed to extend the present method to the 2D case and specify the size, shape and level of symmetry of the generic 2D convolution kernel, $\mathbf{S}$, used for the retrieval of a single wave. A representation of this kernel is provided in Eq. (39) by using tables.

$$
\begin{array}{|l|l|l|}
\hline S_{1,3} & S_{2,3} & S_{3,3} \\
\hline S_{1,2} & S_{2,2} & S_{3,2} \\
\hline S_{1,1} & S_{2,1} & S_{3,1} \\
\hline
\end{array}=\begin{array}{|l|l|l|}
\hline e & c & d \\
\hline b & a & b \\
\hline d & c & e \\
\hline
\end{array}
$$

The symmetry of Eq. (39) $\left(S_{i, j}=S_{j, i}\right)$ mimics that of Eq. (38) where

$$
\forall(i, j) \in \llbracket 1,4 \rrbracket^{2}, \quad \mathbf{D}_{i j}=\mathbf{D}_{j i}^{T},
$$

at least for classical vibroacoustic systems. Both symmetries are also linked to the Hermitian (central) symmetry of the k-space and can be broken by the presence of flow or gyroscopic effects. 
Assuming only one wave type is present in a sequence of $2 \mathrm{D}$ (periodically spaced) perfect measurements $\left(U_{i, j}\right)_{(i, j) \in \llbracket 1, N \rrbracket \times \llbracket 1, M \rrbracket}$, there exists a $2 \mathrm{D}$ convolution operator $\mathbf{S}$ in the form of Eq. (39) such that

$$
\mathbf{V}=\mathbf{U} * \mathbf{S}=\mathbf{0} \text {. }
$$

Or, in a explicit form:

$$
\forall(p, q) \in \llbracket 1, N-3 \rrbracket \times \llbracket 1, M-3 \rrbracket, V_{p, q}=\sum_{(i, j) \in \llbracket 1,3 \rrbracket^{2}} S_{i, j} U_{p+3-i, q+3-j}=0 .
$$

Similarly to the 1D case, it is possible to capture more waves by taking the convolution product of several single wave operators. For two waves, the general form is as follows:

$$
\mathbf{R}=\mathbf{S}_{1} * \mathbf{S}_{2}=\begin{array}{|l|l|l|l|l|}
\hline R_{1,5} & R_{2,5} & R_{3,5} & R_{4,5} & R_{5,5} \\
\hline R_{1,4} & R_{2,4} & R_{3,4} & R_{4,4} & R_{5,4} \\
\hline R_{1,3} & R_{2,3} & R_{3,3} & R_{4,3} & R_{5,3} \\
\hline R_{1,2} & R_{2,2} & R_{3,2} & R_{4,2} & R_{5,2} \\
\hline R_{1,1} & R_{2,1} & R_{3,1} & R_{4,1} & R_{5,1} \\
\hline
\end{array}=\begin{array}{|c|c|c|c|c|}
\hline m & j & g & i & l \\
\hline k & e & c & d & h \\
\hline f & b & a & b & f \\
\hline h & d & c & e & k \\
\hline l & i & g & j & m \\
\hline
\end{array}
$$

As in the 1D case, the coefficients of 2D convolution kernels can be determined using a linear least-squares optimization that reduces to solving an eigenvalue problem. The chain of operations leading to that result is detailed for the generic one wave kernel. First, the matrix $\mathbf{J}_{1}(U)$ is introduced.

$$
\mathbf{J}_{1}(U)=\left(\begin{array}{ccccc}
U_{2,2} & U_{3,2}+U_{1,2} & U_{2,3}+U_{2,1} & U_{3,3}+U_{1,1} & U_{3,1}+U_{1,3} \\
U_{3,2} & U_{4,2}+U_{2,2} & U_{3,3}+U_{3,1} & U_{4,3}+U_{2,1} & U_{4,1}+U_{4,3} \\
\vdots & \vdots & \vdots & \vdots & \vdots \\
U_{i, j} & U_{i+1, j}+U_{i-1, j} & U_{i, j+1}+U_{i, j-1} & U_{i+1, j+1}+U_{i-1, j-1} & U_{i+1, j-1}+U_{i-1, j+1} \\
\vdots & \vdots & \vdots & \vdots & \vdots \\
U_{N-1, M-1} & U_{N, M-1}+U_{N-2, M-1} & U_{N-1, M}+U_{N-1, M-2} & U_{N, M}+U_{N-2, M-2} & U_{N, M-2}+U_{N-2, M}
\end{array}\right) .
$$

The matrix $\mathbf{B}_{1}$, that represents the norm of the $2 \mathrm{D}$ convolution sequence $\mathbf{S}$ as a function of the coefficients' vector is then introduced.

$$
\mathbf{B}_{1}=\left(\begin{array}{ccccc}
1 & 0 & 0 & 0 & 0 \\
0 & 2 & 0 & 0 & 0 \\
0 & 0 & 2 & 0 & 0 \\
0 & 0 & 0 & 2 & 0 \\
0 & 0 & 0 & 0 & 2
\end{array}\right) .
$$

The vector of convolution coefficients, $[a, b, c, d, e]^{T}$, is subsequently obtained by solving the Hermitian eigenvalue problem:

$$
\left(\mathbf{J}_{1}^{*}(U) \mathbf{J}_{1}(U)\right) \mathbf{x}=\rho \mathbf{B}_{1} \mathbf{x} .
$$

Speciffically, the coefficients' vector is the eigenvector associated to the smallest eigenvalue $\rho$ of Eq. (46). This process can easily be generalized to the 2-wave convolution kernel.

\subsection{Extracting wavenumbers and $k$-spaces}

This subsection describes how wavenumbers and k-spaces can be extracted from the convolution kernel of a sequence in a process that mirrors wavenumbers computations in the 2D WFEM framework. We first define $L_{x}$ and $L_{y}$, the sampling periods in the $\mathrm{x}$ and $\mathrm{y}$ directions of the grid and $\mathbf{S}$, the convolution kernel determined in the previous part. A pair of wavenumbers, $\left(k_{x}, k_{y}\right)$, and the associated pair of propagation constants, $\left(\lambda_{x}, \lambda_{y}\right)$, are introduced. They are linked by the relation

$$
\left\{\begin{array}{l}
\lambda_{x}=\mathrm{e}^{-\mathrm{i} k_{x} L_{x}} \\
\lambda_{y}=\mathrm{e}^{-\mathrm{i} k_{y} L_{y}}
\end{array}\right.
$$


We also introduce $\mathbf{V}\left(k_{x}, k_{y}\right)$, the infinite 2D sequence corresponding to the sampling of a plane wave with wavenumbers $\left(k_{x}, k_{y}\right)$ on the regular grid:

$$
\forall(n, m) \in \mathbb{Z}^{2}, \quad V_{n, m}=\lambda_{x}^{n} \lambda_{y}^{m} .
$$

The pair of wavenumbers $\left(k_{x}, k_{y}\right)$ is said to be part of the extended k-space, $K^{e}$ if and only if $\mathbf{V}\left(k_{x}, k_{y}\right) * \mathbf{S}=\mathbf{0}$. In case $\mathbf{S}$ is the generic one wave kernel of Eq. (39), this condition is explicitly developed:

$$
a+b\left(\lambda_{x}+\frac{1}{\lambda_{x}}\right)+c\left(\lambda_{y}+\frac{1}{\lambda_{y}}\right)+d\left(\frac{\lambda_{x}}{\lambda_{y}}+\frac{\lambda_{y}}{\lambda_{x}}\right)+e\left(\lambda_{x} \lambda_{y}+\frac{1}{\lambda_{x} \lambda_{y}}\right)=0
$$

Eq. (49) can be understood as a scalar form of the direct 2D WFEM. As such, it shares the same features. Namely, it can be reduced to a polynomial if either $\lambda_{x}$ or $\lambda_{y}$ (resp. $k_{x}$ or $k_{y}$ ) is fixed. Solutions for the other variable will be found and the pairs will belong to the extended k-space, $K^{e}$. However, these solutions do not have much meaning as it is likely their directions of propagation and directions of decay do not coincide. Forcing this to happen leads to the definition of the proper k-space, $K^{p}$. A pair of wavenumbers, $\left(k_{x}, k_{y}\right)$, belongs to $K^{p}$ if and only if

$$
\exists k \in \mathbb{C}, \exists \theta \in \mathbb{R}, k_{x}=k \cos (\theta), k_{y}=k \sin (\theta), \mathbf{V}\left(k_{x}, k_{y}\right) * \mathbf{S}=\mathbf{0} .
$$

With that supplementary constraint, the directions of propagation and decay are forced to coincide with each other and are given by the angle $\theta$. When $\theta$ is a multiple of $\frac{\pi}{2}$, solving Eq. (49) reduces to finding the roots of a palindromic polynomial as in the $1 \mathrm{D}$ case. To solve for other angles, a continuation method is used whereby the angle $\theta$ is the parameter. The proper k-space, $K^{p}$, can then be retrieved.

\section{Examples}

In this Section, the proposed method is applied to numerical examples. In subsection 4.1, the dispersion curves of an Euler-Bernoulli beam are retrieved by applying the 1D version of INCOME. In subsection 4.2, the wavenumber of the Helmholtz equation is retrieved from a random analytical solution of the equation. Lastly, the dispersion curves and k-space of an orthotropic plate are retrieved using the 2D version of INCOME in subsection 4.3.

\subsection{Euler-Bernoulli Beam}

In this subsection, the $1 \mathrm{D}$ version of INCOME is used to retrieve the dispersion curves of a cantilever EulerBernoulli beam of length $L$ loaded at its end. The system of equations verified by the deflection of the beam, $f(\omega, x)$, is as follows.

$$
\left\{\begin{array}{l}
E I \frac{\partial^{4} f}{\partial x^{4}}-\omega^{2} \zeta f=0 \\
f(\omega, 0)=0, \quad \frac{\partial f}{\partial x}(\omega, 0)=0 \\
\frac{\partial^{2} f}{\partial x^{2}}(\omega, L)=0, \quad-E I \frac{\partial^{3} f}{\partial x^{3}}(\omega, L)=F
\end{array} .\right.
$$

Both the forced response and the dispersion relationship of the beam can be determined analytically from Eq. (51):

$$
k^{4}=\frac{\zeta}{E I} \omega^{2}
$$

The dispersion relation of Eq. (52) describes two pairs of waves. For each pair, one wave propagates in the positive direction while the other propagates in the opposite direction. Additionally, one of these pairs corresponds to strongly evanescent waves which are barely observable. For this numerical example, the values of $F, \zeta$ and $L$ are set to 1 while the product $E I$ is set to $1+0.08 i$. Additionally, the signal's sampling period is chosen equal to $\frac{L}{100}=0.01$, hence, there are 101 samples. First, the 1D INCOME is applied to the displacement fields of Eq. (51) and compared to the analytical solutions of Eq. (52). The retrieved dispersion curves for waves propagating in the positive direction are presented in Figure 1 while the relative error with respect to the analytical solutions is presented in Figure 2.

As can be seen, INCOME achieves results close to numerical precision $\left(2.2 \times 10^{-16}\right)$, which is expected given it is an exact method. However, two phenomena can be observed. Firstly, the relative error for the evanescent waves is 
consistently higher than that of the propagating waves. It makes sense because evanescent waves are barely observable and hardly contribute to the displacement field. Secondly, it can be seen that the error is higher at lower frequencies, which is due to the fact that INCOME has difficulties extracting the correct wavenumbers when the sampling period of the signal is negligible compared to its characteristic wavelength. One way to deal with this issue, instead of changing the measurement period, is to estimate $\lambda^{p}$ instead of $\lambda$. This is equivalent to replacing the matrix $\mathbf{H}_{n_{w}}(U)$ of Eq. (34) with the matrix $\mathbf{H}_{n_{w}, p}(U)$ defined below.

$$
\mathbf{H}_{n_{w}, p}(U)=\left(\begin{array}{ccccc}
U_{2 p n_{w}+1}+U_{1} & U_{p\left(2 n_{w}-1\right)+1}+U_{p+2} & \ldots & U_{p\left(n_{w}+1\right)+1}+U_{p\left(n_{w}-1\right)+1} & U_{p n_{w}+1} \\
U_{2 p n_{w}+2}+U_{2} & U_{p\left(2 n_{w}-1\right)+2}+U_{p+3} & \ldots & U_{p\left(n_{w}+1\right)+2}+U_{p\left(n_{w}-1\right)+2} & U_{p n_{w}+2} \\
\vdots & \vdots & \ldots & \vdots & \vdots \\
U_{N-1}+U_{N-2 p n_{w}-1} & U_{N-p-1}+U_{N-p\left(2 n_{w}-1\right)-1} & \ldots & U_{N-p\left(n_{w}-1\right)-1}+U_{N-p\left(n_{w}+1\right)-1} & U_{N-p n_{w}-1} \\
U_{N}+U_{N-2 p n_{w}} & U_{N-p}+U_{N-p\left(2 n_{w}-1\right)} & \ldots & U_{N-p\left(n_{w}-1\right)}+U_{N-p\left(n_{w}+1\right)} & U_{N-p n_{w}}
\end{array}\right) .
$$

Noteworthy, $\mathbf{H}_{n_{w}, p}(U)$ can also be used when dealing with displacement vectors of size $p$ or periodic structures with $p$ measurement points per period.

In order to test the ability of the method to deal with noise, we add random uncorrelated noise to the solution of Eq. (51) with a signal to noise ratio of 100. Additionally, the formulation described above is used with the matrix $\mathbf{H}_{2, p}$ with $p=10$. The resulting dispersion curves are shown in Figure 3 and the corresponding relative errors in Figure 4. Unsurprisingly, the error trends are similar to that of the noiseless case. The error is higher in the low frequencies and the evanescent waves are more impacted than the propagating ones.

\subsection{Application to the Helmholtz equation}

In this subsection, the 2D INCOME is applied to a solution of the 2D Helmholtz equation with the aim of retrieving its parameter $k$. The equation and its parameter are defined as follows:

$$
\nabla^{2} f+k^{2} f=0 \text {. }
$$

To generate a random valid solution of Eq. (54), punctual sources of random amplitudes, phases and positions are generated outside of the domain of interest. The solution within the domain is then computed analytically since the Green's function $G$ of the equation is known:

$$
G(x, y)=\frac{\mathrm{i}}{4} H_{0}^{(1)}\left(k \sqrt{x^{2}+y^{2}}\right),
$$
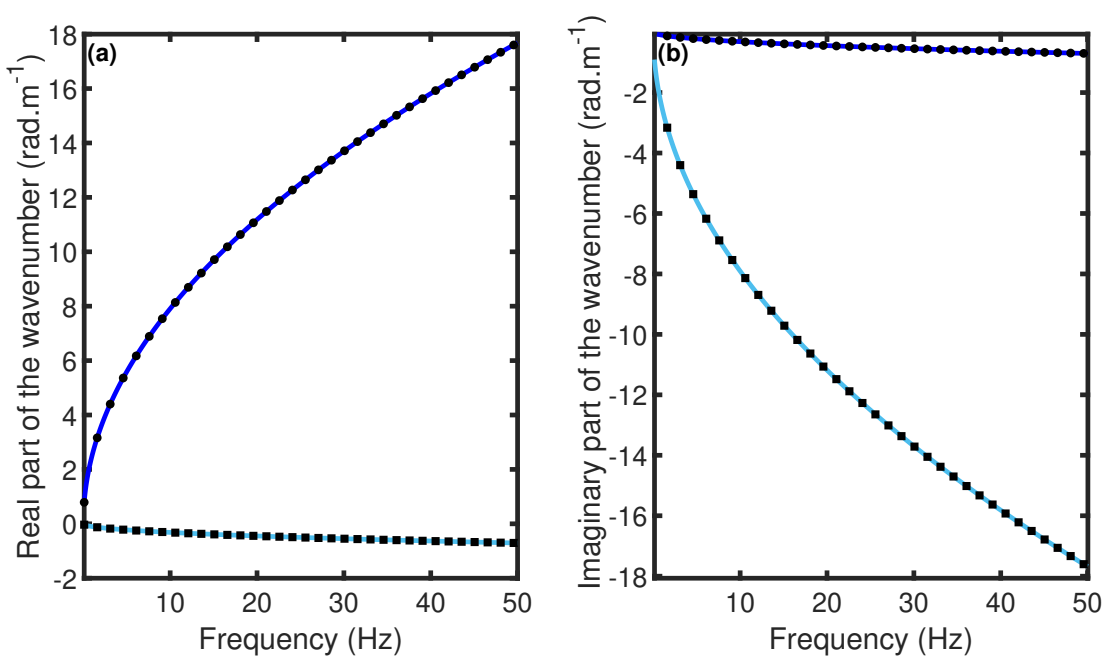

Figure 1: Comparison of the real (a) and imaginary (b) parts of wavenumbers retrieved via the 1D INCOME (solid lines) to analytical solutions (dots and squares). Dark blue lines and dots correspond to the propagating wave while light blue lines and squares correspond to the evanescent wave. 


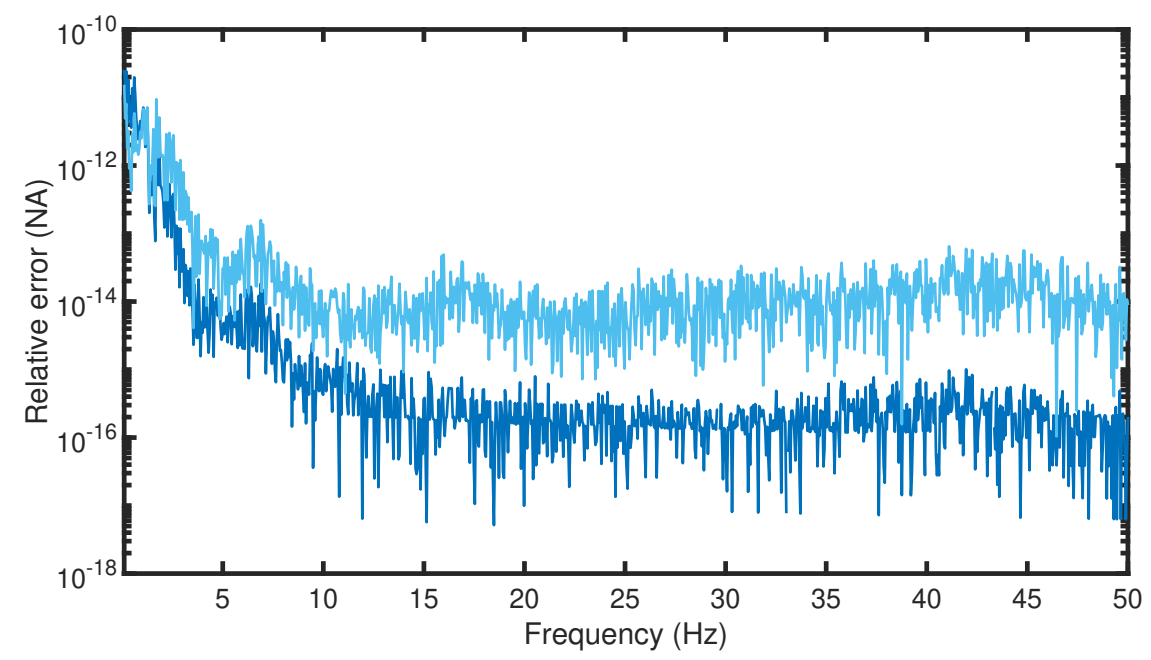

Figure 2: Relative error of INCOME for propagating (dark blue) and evanescent (light blue) waves in the noiseless case.

with $H_{0}^{(1)}$, a Hankel function of the first kind. In view of applying INCOME, the value of $k$ is chosen equal to 30 and a realisation of the random process was generated. The sampling domain was chosen to be a 1 by 1 square centered around the origin, also, the sampling period was set to 0.02 in both directions. Consequently, the sampling grid is a regular 51 by 51 square grid.

Before applying INCOME, the additional symmetries of the Helmholtz equation are accounted for, such that INCOME is used with the following convolution kernel $\mathbf{S}$ instead of the one of Eq. (39):

$$
\begin{array}{|l|l|l|}
\hline S_{1,3} & S_{2,3} & S_{3,3} \\
\hline S_{1,2} & S_{2,2} & S_{3,2} \\
\hline S_{1,1} & S_{2,1} & S_{3,1} \\
\hline
\end{array}=\begin{array}{|l|l|l|}
\hline c & b & c \\
\hline b & a & b \\
\hline c & b & c \\
\hline
\end{array}
$$

Comparing the given value of $k$ with the estimate realised by $\operatorname{INCOME}(\hat{k})$, a relative error $\left|\frac{\hat{k}-k}{k}\right|=5.63 \times 10^{-8}$ is obtained. In order to test the robustness of the method, an uncorrelated complex Gaussian noise is added to the signal such that the signal to noise ratio (SNR) is equal to 10.Using INCOME, an estimate $\hat{k}$ of the wavenumber is retrieved and the corresponding relative error is $\left|\frac{\hat{k}-k}{k}\right|=8.9 \times 10^{-3}$.

\subsection{Application to an orthotropic plate}

In this subsection, the 2D INCOME is applied to the displacement field of a strongly orthotropic thin plate which contains two types of waves (one propagating, one evanescent). The aim is to confirm that INCOME can indeed retrieve the k-space when wave propagation is not isotropic. The dimensions of the thin plate are $0.5 \mathrm{~m}$ by $0.5 \mathrm{~m}$ with a thickness of $3 \mathrm{~mm}$ and its material properties are given in Table 2 and Table 3. The plate is modelled using the Finite Element Method and the modeling is done on ANSYS APDL 17.0 using SHELL181 elements which are based on Mindlin-Reissner plate theory. The mesh is chosen to be a regular 101 by 101 grid. Once the mass and stiffness matrices are extracted, an hysteretic damping $\eta$ of $5 \%$ is added to the model.

\begin{tabular}{|c|c|c|c|c|c|c|c|c|}
\hline Property & Density & $E_{x}$ & $E_{y}$ & $E_{z}$ & $G_{x y}$ & $G_{y z}$ & $G_{x z}$ & $\eta$ \\
\hline Value & $7850 \mathrm{~kg} . \mathrm{m}^{-3}$ & $2.1 \mathrm{GPa}$ & $21 \mathrm{GPa}$ & $210 \mathrm{GPa}$ & $3.033 \mathrm{Gpa}$ & $30.33 \mathrm{Gpa}$ & $2.625 \mathrm{GPa}$ & 0.05 \\
\hline
\end{tabular}

Table 2: Young's Moduli, shear Moduli and hysteretic damping of the plate's material 

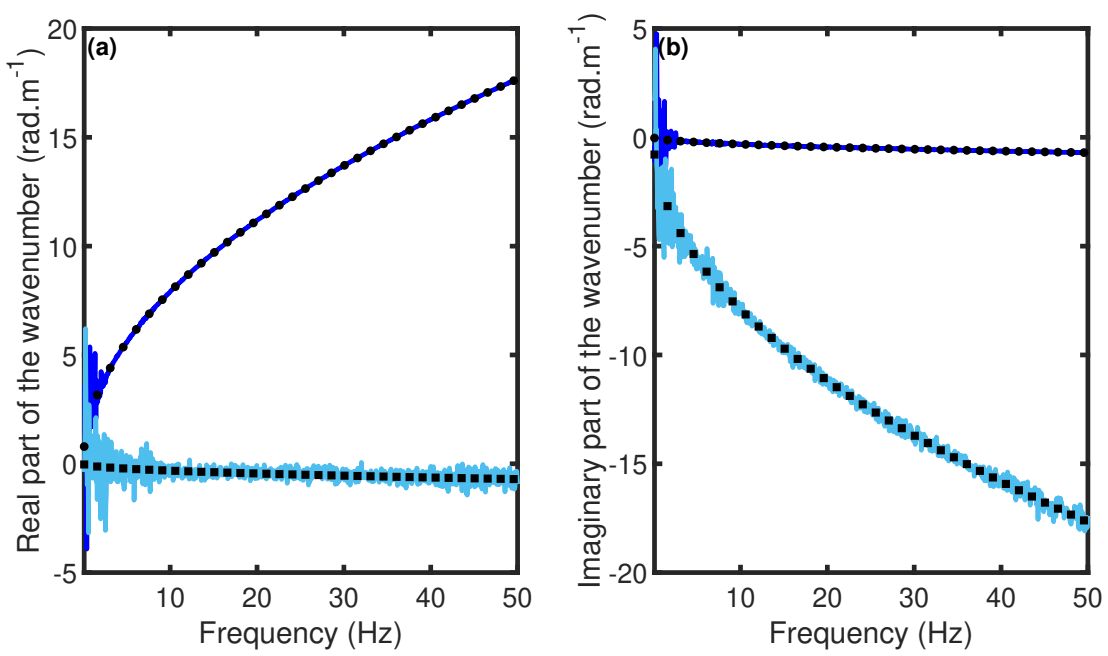

Figure 3: Comparison of the real (a) and imaginary (b) parts of wavenumbers retrieved with INCOME (solid lines) to analytical solutions (dots and square) for a signal to noise ratio of 100. Dark blue lines and dots correspond to the propagating wave while light blue lines and squares correspond to the evanescent wave.

\begin{tabular}{|c|c|c|c|}
\hline Property & $v_{x y}$ & $v_{y z}$ & $v_{x z}$ \\
\hline Value & 0.095 & 0.095 & 0.03 \\
\hline
\end{tabular}

Table 3: Poisson's ratios of the plate materia

Accounting for the propagation symmetries of the orthotropic plate, the convolution kernel of Eq. (43) is replaced by the following one.

$$
\mathbf{R}=\begin{array}{|l|l|l|l|l|}
\hline R_{1,5} & R_{2,5} & R_{3,5} & R_{4,5} & R_{5,5} \\
\hline R_{1,4} & R_{2,4} & R_{3,4} & R_{4,4} & R_{5,4} \\
\hline R_{1,3} & R_{2,3} & R_{3,3} & R_{4,3} & R_{5,3} \\
\hline R_{1,2} & R_{2,2} & R_{3,2} & R_{4,2} & R_{5,2} \\
\hline R_{1,1} & R_{2,1} & R_{3,1} & R_{4,1} & R_{5,1} \\
\hline
\end{array}=\begin{array}{|l|l|l|l|l|}
\hline i & g & f & g & i \\
\hline h & d & c & d & h \\
\hline e & b & a & b & e \\
\hline h & d & c & d & h \\
\hline i & g & f & g & i \\
\hline
\end{array}
$$

First, the frequency forced response of the plate for a point force excitation at the location $(x, y)=(0,0.25)$ is computed on the $0 \mathrm{~Hz}-300 \mathrm{~Hz}$ frequency range. Free boundary conditions are used. The dispersion curves in the main sampling directions and the $\mathrm{k}$-space at $300 \mathrm{~Hz}$ are extracted from the computed displacement fields using the 2D INCOME. To provide some comparison, numerical dispersion curves are computed using the Shift-Cell Operator Method [24] -a numerical method similar to the WFEM that enables the computations of k-spaces in damped structures- with a unit cell containing 20 quadratic elements in the thickness of the plate. The results obtained with each method are presented in Figure 5 and Figure 6. There is good agreement between both techniques and differences should be attributed to the difference in the underlying theories used for the modeling. Indeed, dispersion curves from INCOME were recovered from displacement fields obtained by a discretization of the Mindlin-Reissner theory. By contrast, the Shift-Cell Method accounts for all 3D effects, some of which cannot be completely neglected due to the strongly orthotropic material properties. Nonetheless, one would be hard pressed to tell the difference between the k-spaces in Figure 6, which demonstrates the ability of INCOME to extract coherent continuous k-spaces from input wavefields.

\section{Conclusion}

In this paper, a method for one-dimensional and two-dimensional wavenumber extraction is presented. Based on a convolution framework, the method requires periodic sampling of the signal of interest to produce a convolution kernel that describes its wavenumbers or k-space. The core of INCOME is similar to the Prony series method but 


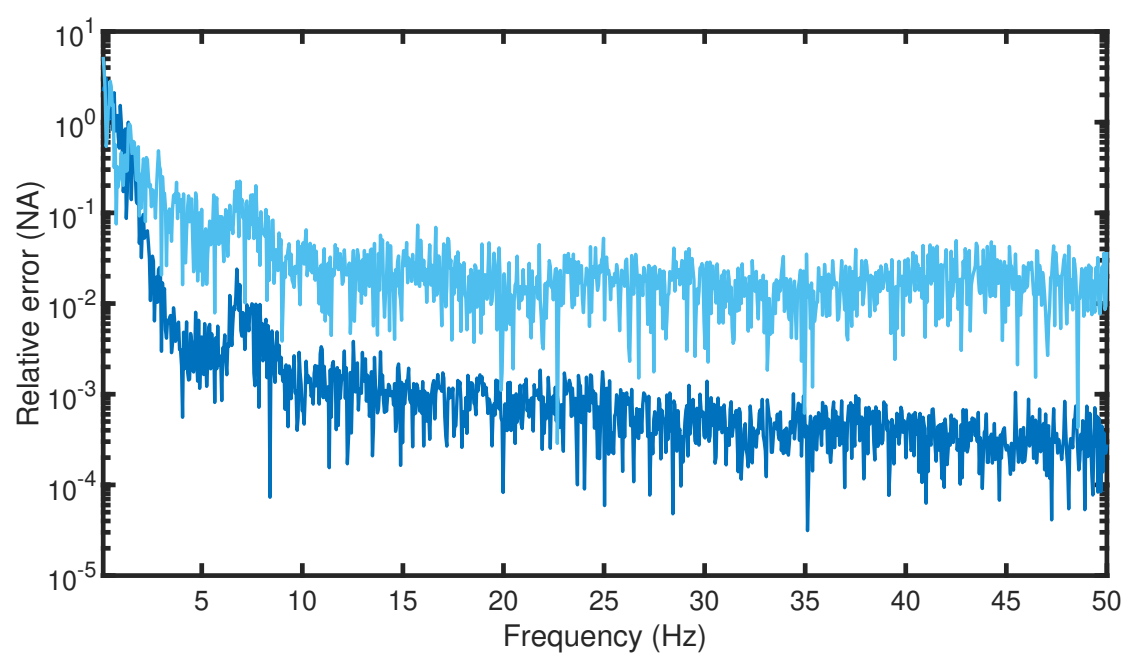

Figure 4: Relative error of INCOME for propagating (dark blue) and evanescent (light blue) waves for a signal to noise ratio of 100.
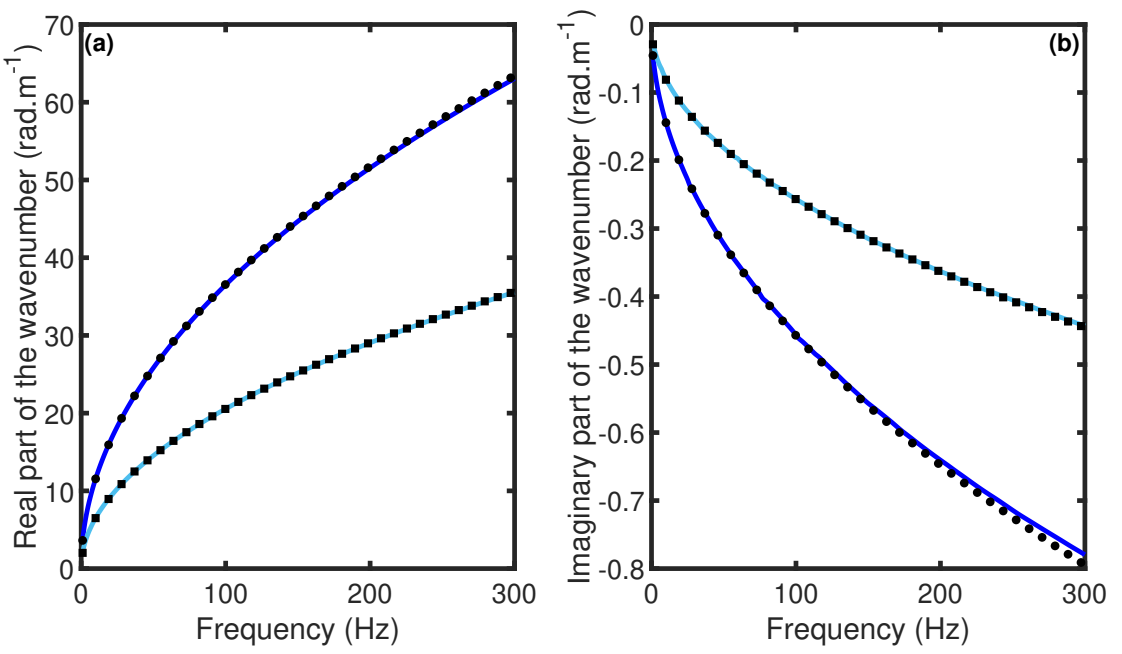

Figure 5: Comparison of the dispersion curves in the main directions of the orthotropic plate computed via the Shift-Cell Operator method (dots and squares) or retrieved with INCOME (solid lines).(a) real parts of the wavenumber. (b) imaginary parts of the wanumbers. Dark blue lines and dots are used for the $\mathrm{X}$ direction, light blue lines and squares are used for the $\mathrm{Y}$ direction.

takes advantage of a convolution framework to encode wave propagation symmetries and reach the $2 \mathrm{D}$ case. The main conclusions of the paper achievements are summarised as follows:

- This paper provides a novel formulation trying to address some of the issues connected to existing wavenumbers extraction methods. The paper also gives a comprehensive state of the art of most published techniques and methods aiming at k-space identification through an inverse way of thinking. This state of the art positioning is, in the authors' opinion, a concise guideline for bench-marking and for further developments.

- The INCOME method relies on solving small linear problems and is exact in both 1D and 2D cases. The wording "exact" refers here to the propagation scheme adopted for the inverse algorithm. Precisely, a given propagation model is fitted to the input data with consistency. The INCOME method is also mathematically robust and its numerical implementation easy to handle. 
(a)

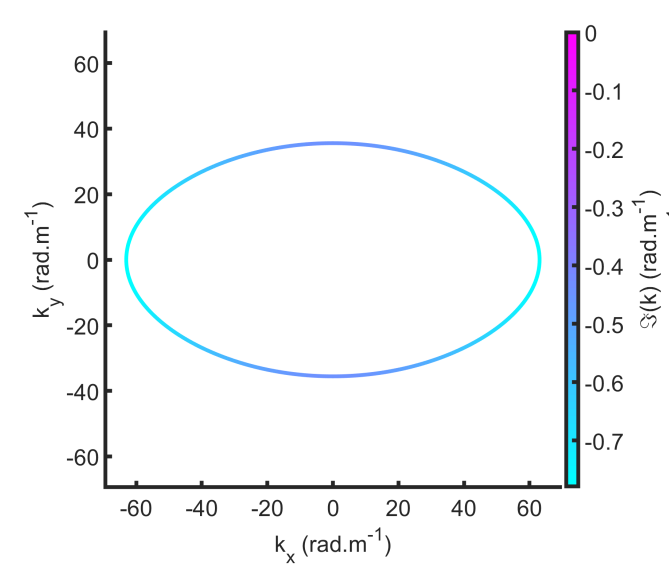

(b)

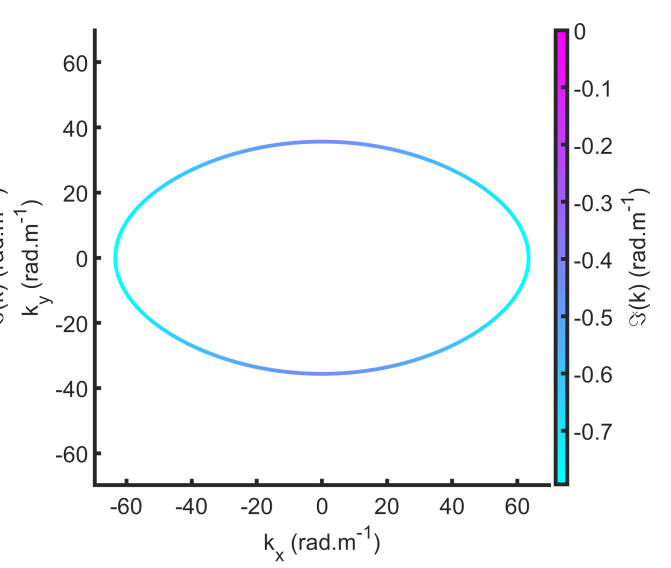

Figure 6: k-space at $300 \mathrm{~Hz}$ for the propagating waves of the orthotropic plate retrieved via INCOME (a) and computed via the Shift Cell Operator method (b).

- In the $2 \mathrm{D}$ case, all properties of the $\mathrm{k}$-space are retrieved together in a coherent manner and INCOME was shown to successfully identify the wave heading (directivity) and the spatial attenuation. This is the most advantageous property of INCOME and it is achieved by making periodic sampling of the input mandatory.

- The proposed method draws inspiration from the WFEM framework [14-17, 25] and can be considered an inverse WFEM approach. The periodicity requirement for INCOME and the use of recurrence relationships make the formulation comprehensive for WFEM practitioners. The literature belonging to the WFEM being rich, one could expect bridges to be created between the direct WFEM and the INCOME.

Finally, the accuracy of the proposed method was demonstrated in both 1D and 2D scenarios and it was shown to be somewhat noise resistant. Nonetheless, it is expected that this aspect of the method could be improved by applying regularization techniques similar to those used for the Prony series method. Also, incorporating metadata (e.g. coherence data for structural measurements) in the formulation could further improve its robustness.

\section{Acknowledgements}

The research of R. F. Boukadia is funded by an Early Stage Researcher grant within the European Project VIPER Marie Curie Initial Training Network (GA 675441). C. Droz is an individual Marie Skłodowska-Curie fellow funded by the European Union's Horizon 2020 research and innovation programme under the grant agreement No. 797034 (WIDEA). The research of E. Deckers is funded by a grant from the Research Foundation - Flanders (FWO). The work leading to this publication has been funded by the ICON project "DETECT-ION", which fits in the MacroModelMat (M3) research program, coordinated by Siemens (Siemens Digital Industries Software, Belgium) and funded by SIM (Strategic Initiative Materials in Flanders) and VLAIO (Flemish government agency Flanders Innovation \& Entrepreneurship).

\section{Appendix A. Deriving Eq. (38)}

In this section, Eq. (38) is briefly derived. For the sake of simplicity, the derivation is restricted to the case a 2D homogeneous medium but a more thorough derivation compatible with periodic media was presented in [23]. First, the unit cell (UC) of the periodic media is considered and discretized using, e.g., the finite element method. The 
corresponding stiffness and masss matrices, $\mathbf{K}$ and $\mathbf{M}$, are thus available. Since the medium is homogeneous, the UC can be disctretized such that it has no inner or side degrees of freedom (dofs). The displacment vector of the $\mathrm{UC}, \mathbf{U}$, can thus be expressed as a function of all its corner dofs $\mathbf{q}_{1}, \mathbf{q}_{2}, \mathbf{q}_{3}$ and $\mathbf{q}_{4}$ (defined in Figure A.7) such that $\mathbf{U}^{T}=\left[\mathbf{q}_{1}^{T}, \mathbf{q}_{2}^{T}, \mathbf{q}_{3}^{T}, \mathbf{q}_{4}^{T}\right]$.

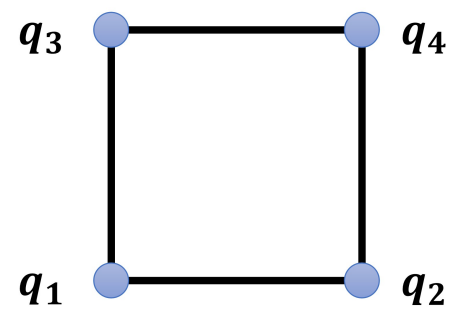

Figure A.7: Schematic representation of the UC of a 2D homogenous medium and definition of is corner's dofs $q_{1}, q_{2}, q_{3}$ and $q_{4}$.

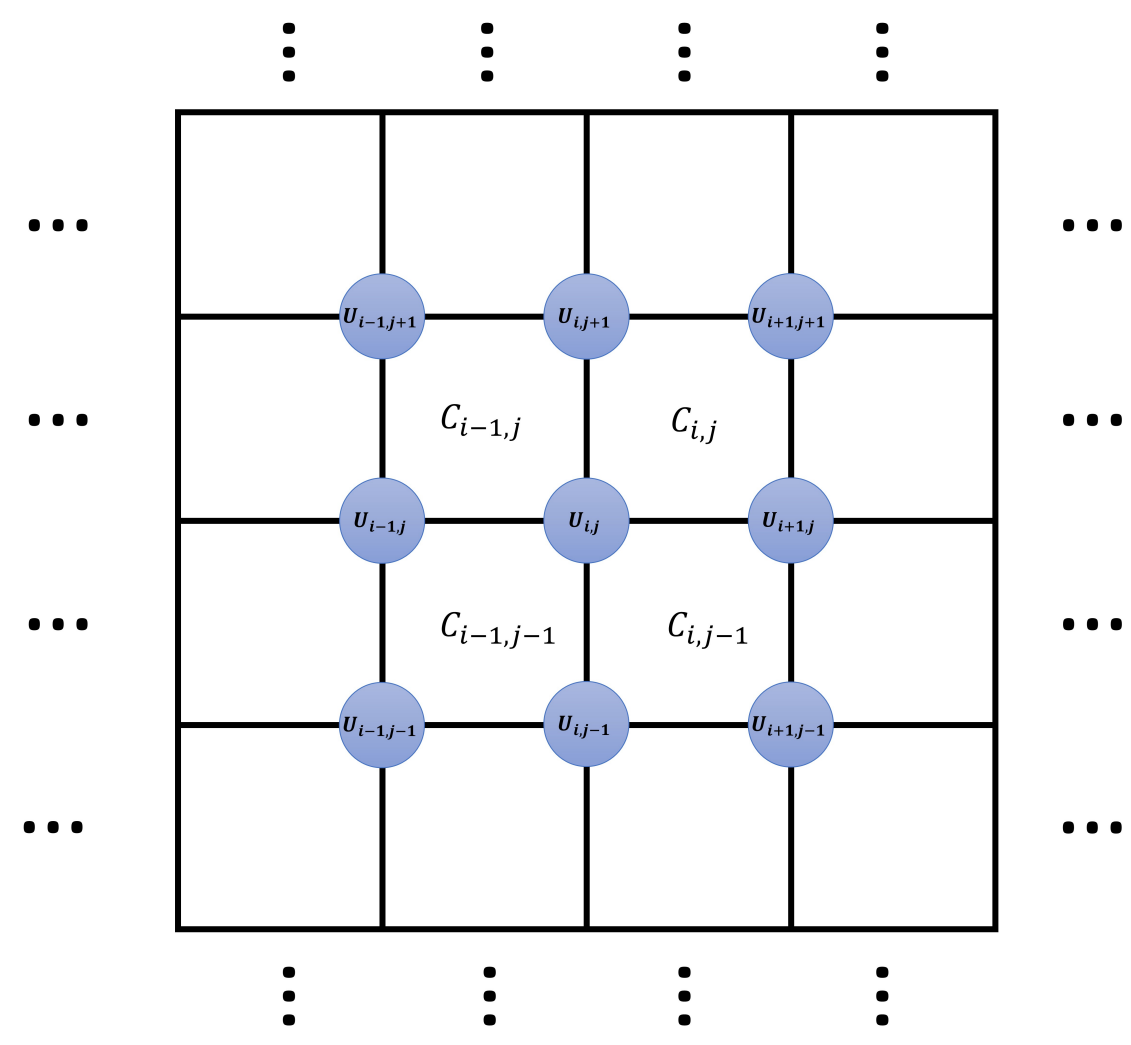

Figure A.8: Representation of a 2D infinite homogeneous medium as an assembly of unit cells $\left(C_{p, q}\right)$ with displacement vectors $\left(U_{p, q}\right)$.

In order to describe the dynamics of the $2 \mathrm{D}$ infinite periodic medium, an infinite assembly of identical UCs is realized according to Figure A.8 where $C_{i, j}$, the UC numbered $(i, j)$, has corner dofs $\mathbf{q}_{1}=\mathbf{U}_{i, j}, \mathbf{q}_{2}=\mathbf{U}_{i+1, j}, \mathbf{q}_{3}=\mathbf{U}_{i, j+1}$ and $\mathbf{q}_{4}=\mathbf{U}_{i+1, j+1}$.

Considering a circular frequency $\omega$, the dynamic striffness matrix $\mathbf{D}=\mathbf{K}-\omega^{2} \mathbf{M}$ of the UC is defined. In order to 
ease the following developments, $\mathbf{D}$ is partitioned according to the UC's sparial structure such that the UC's dynamical equation is written as

$$
\left[\begin{array}{llll}
\mathbf{D}_{11} & \mathbf{D}_{12} & \mathbf{D}_{13} & \mathbf{D}_{14} \\
\mathbf{D}_{21} & \mathbf{D}_{22} & \mathbf{D}_{23} & \mathbf{D}_{24} \\
\mathbf{D}_{31} & \mathbf{D}_{32} & \mathbf{D}_{33} & \mathbf{D}_{34} \\
\mathbf{D}_{41} & \mathbf{D}_{42} & \mathbf{D}_{43} & \mathbf{D}_{44}
\end{array}\right]\left[\begin{array}{l}
\mathbf{q}_{1} \\
\mathbf{q}_{2} \\
\mathbf{q}_{3} \\
\mathbf{q}_{4}
\end{array}\right]=\left[\begin{array}{l}
\mathbf{F}_{1} \\
\mathbf{F}_{2} \\
\mathbf{F}_{3} \\
\mathbf{F}_{4}
\end{array}\right]
$$

with $\mathbf{F}_{1}, \mathbf{F}_{2}, \mathbf{F}_{3}$ and $\mathbf{F}_{4}$ forces applied at the corresponding corners of the UC. With everything defined, the equation of motion of the infinite medium can be derived. For any pair of integers $(i, j)$, the dynamical equilibrium at the corresponding corner is obtained by considering the summed contributions of the cells $C_{i, j}, C_{i-1, j}, C_{i, j-1}$ and $C_{i-1, j-1}$ at their shared dofs vector $\mathbf{U}_{i, j}$. First, the contribution of each cell is detailed,

$$
\left\{\begin{aligned}
C_{i, j} & \longrightarrow \mathbf{D}_{11} \mathbf{U}_{i, j}+\mathbf{D}_{12} \mathbf{U}_{i+1, j}+\mathbf{D}_{13} \mathbf{U}_{i, j+1}+\mathbf{D}_{14} \mathbf{U}_{i+1, j+1} \\
C_{i-1, j} & \longrightarrow \mathbf{D}_{21} \mathbf{U}_{i-1, j}+\mathbf{D}_{22} \mathbf{U}_{i, j}+\mathbf{D}_{23} \mathbf{U}_{i-1, j+1}+\mathbf{D}_{24} \mathbf{U}_{i, j+1} \\
C_{i, j-1} & \longrightarrow \mathbf{D}_{31} \mathbf{U}_{i, j-1}+\mathbf{D}_{32} \mathbf{U}_{i+1, j-1}+\mathbf{D}_{33} \mathbf{U}_{i, j}+\mathbf{D}_{34} \mathbf{U}_{i+1, j} \\
C_{i-1, j-1} & \longrightarrow \mathbf{D}_{41} \mathbf{U}_{i-1, j-1}+\mathbf{D}_{42} \mathbf{U}_{i, j-1}+\mathbf{D}_{43} \mathbf{U}_{i-1, j}+\mathbf{D}_{44} \mathbf{U}_{i, j}
\end{aligned}\right.
$$

and the dynamical equation of the waveguide is derived be equating the summed contributions to $F_{i, j}$, the vector of external forces acting on the medium at the corner $(i, j)$.

$$
\begin{aligned}
\mathbf{F}_{i, j}= & \left(\mathbf{D}_{11}+\mathbf{D}_{22}+\mathbf{D}_{33}+\mathbf{D}_{44}\right) \mathbf{U}_{i, j}+ \\
& \left(\mathbf{D}_{13}+\mathbf{D}_{24}\right) \mathbf{U}_{i, j+1}+\left(\mathbf{D}_{12}+\mathbf{D}_{34}\right) \mathbf{U}_{i+1, j}+\left(\mathbf{D}_{31}+\mathbf{D}_{42}\right) \mathbf{U}_{i, j-1}+\left(\mathbf{D}_{21}+\mathbf{D}_{43}\right) \mathbf{U}_{i-1, j}+. \\
& \mathbf{D}_{14} \mathbf{U}_{i+1, j+1}+\mathbf{D}_{23} \mathbf{U}_{r-1, s+1}+\mathbf{D}_{32} \mathbf{U}_{r+1, s-1}+\mathbf{D}_{41} \mathbf{U}_{r-1, s-1}
\end{aligned}
$$

Eq. (38) is finally retrieved.

\section{References}

[1] A. M. Ibrahim, M. I. Marei, S. F. Mekhamer, M. M. Mansour, An artificial neural network based protection approach using total least square estimation of signal parameters via the rotational invariance technique for flexible AC transmission system compensated transmission lines, Electric Power Components and Systems 39 (2011) 64-79.

[2] L. Van Belle, C. Claeys, E. Deckers, W. Desmet, On the impact of damping on the dispersion curves of a locally resonant metamaterial: Modelling and experimental validation, Journal of Sound and Vibration 409 (2017) 1-23.

[3] J. Zhang, E. Reynders, G. De Roeck, G. Lombaert, Model updating of periodic structures based on free wave characteristics, Journal of Sound and Vibration 442 (2019) 281-307.

[4] G. Tufano, F. Errico, O. Robin, C. Droz, M. Ichchou, B. Pluymers, W. Desmet, N. Atalla, K-space analysis of complex large-scale metastructures using the Inhomogeneous Wave Correlation method, Mechanical Systems and Signal Processing 135 (2020) 106407.

[5] R. Kumaresan, D. W. Tufts, Estimating the Parameters of Exponentially Damped Sinusoids and Pole-Zero Modeling in Noise, IEEE Transactions on Acoustics, Speech, and Signal Processing 30 (1982) 833-840.

[6] A. Roy, A. Paulraj, T. Kailath, Estimation of Signal Parameters Via Rotational Invariance Techniques-Esprit., Proceedings - IEEE Military Communications Conference (1986).

[7] J. G. McDaniel, W. S. Shepard, Estimation of structural wave numbers from spatially sparse response measurements, The Journal of the Acoustical Society of America 108 (2002) 1674-1682.

[8] J. Berthaut, M. N. Ichchou, L. Jezequel, K-space identification of apparent structural behaviour, Journal of Sound and Vibration 280 (2005) $1125-1131$.

[9] C. R. Halkyard, Maximum likelihood estimation of flexural wavenumbers in lightly damped plates, Journal of Sound and Vibration 300 (2007) 217-240.

[10] G. Chardon, A. Leblanc, L. Daudet, Plate impulse response spatial interpolation with sub-Nyquist sampling, Journal of Sound and Vibration 330 (2011) 5678-5689.

[11] P. Margerit, A. Lebée, J. F. Caron, K. Ege, X. Boutillon, The High-Resolution Wavevector Analysis for the characterization of the dynamic response of composite plates, Journal of Sound and Vibration 458 (2019) 177-196.

[12] P. Cull, M. Flahive, R. Robson, Difference Equations, Undergraduate Texts in Mathematics, Springer-Verlag, New York, 2005.

[13] B. Van Damme, A. Zemp, Energy distribution and exchange between spatial harmonics in bending wave phononic crystals, Phys. Rev. Applied 10 (2018) 014001.

[14] J.-M. Mencik, M. Ichchou, Multi-mode propagation and diffusion in structures through finite elements, European Journal of MechanicsA/Solids 24 (2005) 877-898.

[15] D. J. Mead, The forced vibration of one-dimensional multi-coupled periodic structures: An application to finite element analysis, Journal of Sound and Vibration 319 (2009) 282-304. 
[16] T. Gras, M. A. Hamdi, M. Ben Tahar, O. Tanneau, L. Beaubatie, On a coupling between the Finite Element (FE) and the Wave Finite Element (WFE) method to study the effect of a local heterogeneity within a railway track, Journal of Sound and Vibration 429 (2018) 45-62.

[17] N. S. Bardell, D. J. Mead, Free vibration of an orthogonally stiffened cylindrical shell, part I: Discrete line simple supports, Journal of Sound and Vibration 134 (1989) 29-54.

[18] W. X. Zhong, F. W. Williams, on the Direct Solution of Wave-Propagation for Repetitive Structures, Journal of Sound and Vibration 181 (1995) 485-501.

[19] O. Roy, M. Vetterli, The effective rank: A measure of effective dimensionality, European Signal Processing Conference (2007) 606-610.

[20] E. Fishler, H. V. Poor, Estimation of the number of sources in unbalanced arrays via information theoretic criteria, IEEE Transactions on Signal Processing 53 (2005) 3543-3553. arXiv: 0501058.

[21] S. Akrout, Comportement dynamique déterministe et large bande des structures guidées, Ph.D. thesis, Ecully, Ecole centrale de Lyon, 2005.

[22] B. R. Mace, E. Manconi, Modelling wave propagation in two-dimensional structures using finite element analysis, Journal of Sound and Vibration 318 (2008) 884-902.

[23] R. Boukadia, Projet VIPER: Modélisation de structures périodiques dans le cadre d'applications vibroacoustiques [Modelling of periodic structures for vibroacoustic applications], Master's thesis, École Centrale de Lyon, 2016.

[24] M. Collet, M. Ouisse, M. Ruzzene, M. Ichchou, Floquet-bloch decomposition for the computation of dispersion of two-dimensional periodic, damped mechanical systems, International Journal of Solids and Structures 48 (2011) 2837 - 2848.

[25] Y. Fan, M. Collet, M. Ichchou, L. Li, O. Bareille, Z. Dimitrijevic, Energy flow prediction in built-up structures through a hybrid finite element/wave and finite element approach, Mechanical Systems and Signal Processing 66-67 (2016) 137 - 158. 